\title{
What to believe about your belief that you're in the good case
}

\author{
Alex Worsnip
}

\author{
Forthcoming in Oxford Studies in Epistemology, vol. 6 \\ Penultimate version; please cite published version if possible
}

Going about our daily lives in an orderly manner requires us, once we are aware of them, to dismiss many metaphysical possibilities. We take it for granted that we are not brains in vats, or living in the Matrix, or in an extended dream. Call these things that we take for granted "anti-skeptical assumptions". They can be so-called because such apparent possibilities, famously, are pressed into action by skeptics as threatening vast swathes of (what we take to be) our ordinary knowledge. But one need not be impressed by such arguments against knowledge to be interested in the epistemic status of such anti-skeptical assumptions. We do appear to take it for granted in our belief-forming practices that appearances are some kind of guide to reality; that appearance and reality are not, as they are in the skeptical scenarios, radically out of sync. We assume that we are "in the good case", such that things are roughly as they seem to be.

In talking of our mental states with respect to such propositions as 'assumptions', I do not mean to be putting any special weight on that term, or to be using it in a way that marks assumptions off from beliefs as a sui generis mental state. Indeed, I think it is plausible that many of us do (at least implicitly) believe such propositions. ${ }^{1}$ Even if this is not always an explicit belief, it can be made explicit. And we can ask: what should a reflective agent then think of such a belief?

For most ordinary beliefs we hold, we take ourselves to have some kind of evidence for the belief in question. More cautiously, there is something odd about holding a belief in the face of a considered judgment that one lacks adequate evidence for that belief. If someone says, "I have no evidence whatsoever that penguins fly - nevertheless, penguins do in fact fly", it is hard to get a grip on how they are best interpreted. It's natural to wonder, for example, whether some narrow or idiosyncratic conception of evidence is at work. It's puzzling why someone who themselves thought there was no evidence whatsoever that penguins fly would nevertheless think that penguins do fly. If that really is the right description of the agent's mental states, she seems to be guilty of a particular kind of irrationality that amounts to internal incoherence.

Yet, as we'll see, it can be puzzling just what our evidence for anti-skeptical assumptions could be. So some philosophers think that such beliefs can or even must be held without evidence - and, indeed, in the face of an acknowledgment on the part of the believing agent that she lacks evidence for them. Regardless of one's attitude toward philosophical skepticism, it is interesting to ask whether this is so, and if so how to fit that into our general epistemological theories about rationality, evidence,

For helpful discussions, I'm grateful to audiences at a workshop at Union College and at a meeting of the Society for Skeptical Studies at the 2017 Eastern APA, and to David James Barnett, Julianne Chung, Justin Clarke-Doane, Sophie Horowitz, Jessie Munton, Ram Neta, Kate Nolfi, John Pittard, Jim Pryor, and two anonymous referees for OSE. Special thanks to Keith DeRose for his encouragement of this project and for comments and discussions on these topics over a number of years.

${ }^{1}$ If you think many of us don't count as fully believing such things, you can still consider an agent that does have such a belief. I will consider the proposal that we are warranted in assuming, but not believing, such propositions - where assumption is sharply distinguished from belief - in section III(b) below. 
and coherence. My ultimate aim will be to argue that anti-skeptical assumptions do not constitute an exception to the principle that it is irrational to hold a belief that one actively takes oneself to lacks adequate evidence for. But I will aim to preserve this result while also doing justice to the considerations that motivate thinking of anti-skeptical assumptions as somehow evidentially groundless.

\section{Evidence and inter-level coherence}

We should distinguish two distinct claims that might be challenged by a view of anti-skeptical assumptions as evidentially groundless. First, there is the claim that epistemically ideal agents never believe anything without (sufficient) evidence. Call this the "evidential requirement". Second, there is the claim that epistemically ideal agents never believe something which they themselves judge themselves to lack sufficient evidence for. In other words, their first-order and higher-order beliefs display a distinctive kind of coherence, satisfying the following principle:

Inter-level coherence (ILC). Rationality requires of you that if you believe that you lack sufficient evidential support for some doxastic attitude $\mathrm{D}(\mathrm{p})$, then you don't take $\mathrm{D}(\mathrm{p})$.

(ILC) is a coherence requirement, in the sense that it governs which combinations of attitudes a rational agent may hold jointly together. Following many theorists of coherence requirements, ${ }^{2}$ I formulate it with "wide scope": the 'rationality requires' operator takes scope over the whole conditional that follows it, which is stipulatively to be read as the material conditional. One violates (ILC) just if one simultaneously believes that one lacks adequate evidence for some doxastic attitude $\mathrm{D}(\mathrm{p})$ and yet also takes that attitude. ${ }^{3}$

The relationship between the evidential requirement and (ILC) is complex. Some believe them to be mutually supporting; others believe them to stand in some tension with each other. Neither of these views is obvious without argument. I have written about this relationship at length elsewhere; ${ }^{4}$ here it suffices to say that, since (ILC) is wide-scope, it doesn't simply give one's higher-order beliefs about what one's evidence supports a fixed authority over what one is rationally required to believe. Rather, it treats one's higher-order beliefs as just as revisable as one's first-order beliefs. So it won't straightforwardly conflict with the evidential requirement, every time one is mistaken about what one's evidence supports.

The challenge from anti-skeptical assumptions, in its most robust form, is plausibly viewed as a challenge to both of these principles. For much of what follows, I will be simply talking about

\footnotetext{
2 See, e.g., Broome (1999, 2013: ch. 8); Dancy (1977); Scanlon (2007).

${ }^{3}$ Some (e.g. Horowitz 2014) call inter-level coherence an "anti-akratic" principle. I eschew this label for two reasons. First, my chosen formulation of inter-level coherence refers to one's higher-order judgments about what one's evidence supports, not to one's higher-order judgments about what one ought to believe. Secondly, there are rationales for interlevel coherence that do not turn on any appeal to the idea that an agent who violates it is akratic or weak-willed, and that find no analogue in cases of practical akrasia.

4 Worsnip (forthcoming-a).
} 
whether we really have evidence for anti-skeptical assumptions. But it would be somewhat odd, if potentially interesting, to say that while we cannot in fact have such evidence, ideal agents are precluded from ever realizing this. Given that, the idea that we cannot ever have evidence for antiskeptical assumptions will also be a problem for (ILC). Later on, (ILC) will play an important role in my positive account of how to understand and treat anti-skeptical assumptions.

There are, needless to say, other challenges to (ILC), unrelated to anti-skeptical assumptions. I deal with those challenges elsewhere. ${ }^{5}$ Here I am focused solely on the challenge from anti-skeptical assumptions.

\section{Naïve evidentialism}

Here is a major source of worry about whether we can have evidence for anti-skeptical assumptions. Effective skeptical scenarios are designed such that for any candidate piece of evidence for an antiskeptical assumption, the skeptical scenario predicts that you have that evidence. So, for example, it appears to you that you have hands; but a suitably refined brain-in-a-vat hypothesis predicts that it appears that you have hands. For any potential candidate piece of evidence against the skeptical hypothesis, some additional detail can be built into the skeptical hypothesis to predict that you will have that evidence even when the skeptical scenario obtains. Given this, it is tempting to think that none of these candidate pieces of evidence can in fact be evidence for the anti-skeptical assumptions.

One might react to this worry in a number of ways. The first response I want to consider says that it simply doesn't matter that the skeptical hypothesis predicts that you have the evidence in question; nevertheless, the evidence can be perfectly good evidence for anti-skeptical assumptions that deny that hypothesis. ${ }^{6}$ Thus, ordinary appearances can serve as evidence for anti-skeptical assumptions. This strategy promises to deliver the result that we have evidence for the anti-skeptical assumption whether this assumption in fact obtains or not - that is, both in the good case and in the bad case: either way, the appearance of hands supports the good case hypothesis over the bad case hypothesis. This is an advantage insofar as one has internalist inclinations. ${ }^{7}$ Let us call this strategy "naïve evidentialism" about anti-skeptical assumptions. ${ }^{8}$ I will offer two broad sets of reasons for doubting naïve evidentialism.

\section{(a) Evidential parity}

Let's begin with some of the cases that defenders of the naïve evidentialist view have given in an attempt to warm us up to the idea that ordinary appearances can provide evidence for anti-skeptical assumptions. Here is one due to Ned Markosian (2014: 170, 172):

\footnotetext{
${ }^{5}$ Ibid.

${ }^{6}$ See, e.g., Conee \& Feldman (2004), Markosian (2014), and perhaps Steup (2011). It is also natural to think of Pryor's (2000) "dogmatist" view as congenial to this position, but it is worth noting that his primary focus is on what justifies us in believing ordinary hypotheses (e.g.: I have hands), not on what justifies us in believing the anti-skeptical assumptions that these ordinary hypotheses entail. See Pryor (2000: 546, n. 35) for helpful clarification.

7 Where internalism is understood as entailing that one's justification in the good and the bad case must identical. ${ }^{8}$ I call it 'naïve' not as an insult but to mark the difference with a more complex evidentialist view on which one's evidence crucially differs across the good case and the bad case. I will turn to such externalist accounts in section IV.
} 
Blackjack. Suppose you're playing blackjack and have been counting the cards. You know that there are 10 cards left in the deck, and that 9 of them are face cards.

Markosian, reasonably, claims that in this case the evidence supports the hypothesis that the next card drawn will be a face card. But now consider the alternative hypothesis that even though nine out of the ten cards are face cards, the next card drawn will be the only remaining number card. It's built into this hypothesis that nine out of the ten cards are face cards, so this hypothesis predicts the evidence you have. Yet, Markosian contends, the evidence still seems to (at least pro tanto) support the hypothesis that the next card drawn will be a face card, and not the proposition that the next card drawn will be the only remaining number card of the ten total cards remaining.

Markosian gives several other similar cases. You see what appears to be a car parked on the street, and this evidence supports the common-sense hypothesis that your empty car is parked on the street, not the alternative hypothesis that a car filled with a dozen cleverly hidden clowns is parked on the street (Markosian 2014: 171). You see someone perform an (apparently) very kind act, and your evidence supports the common-sense hypothesis that this person is a nice person, not the alternative hypothesis that this person is the devil calculatedly appearing nice for a nefarious reason (ibid: 1701). ${ }^{9}$ In all of these cases, were the alternative hypothesis to obtain, one would have the same evidence that one in fact has; yet Markosian holds that the evidence still supports the common-sense hypothesis rather than the alternative one. Markosian contends that we should say the same thing about ordinary hypotheses as compared to skeptical ones.

How much of the description that Markosian and Steup offer is non-negotiable? To have a single case to work with, I will focus on the blackjack case (which nicely gives us numbers to work with when describing evidential probabilities), but what I say could be adapted to any of the other cases. Let 'NextF' be the proposition that the next card drawn will be a face card, and '9/10RemainingF' be the proposition that 9 of the 10 remaining cards are face cards. Against the background of the evidence that 9/10RemainingF, NextF is the analogue of the "ordinary" or "common-sense" hypothesis here. Recall that the contrasting "alternative" hypothesis, analogous to a skeptical hypothesis, that Markosian wants us to consider is that even though nine of the ten remaining cards are face cards, the next card is a number card. For the sake of simplicity, let's treat it as axiomatic that any card that is not a face card is a number card. Given that, the alternative hypothesis, analogous to the skeptical hypothesis, is equivalent to ( $/ / 10$ RemainingF \& $\neg \mathrm{NextF})$. The analogous proposition to the anti-skeptical assumption, then, is $\neg(9 / 10$ RemainingF \& $\neg \mathrm{NextF})$. Note that this is not equivalent to the proposition NextF, just as the hypothesis that one is not a handless brain-in-a-vat is not equivalent to the proposition that one has hands.

Markosian is surely right that 9/10RemainingF does (at least pro tanto) support NextF. But our interest is not (directly) in NextF. We are interested in anti-skeptical assumptions, and as I've just said, the analogue of the anti-skeptical assumption here is $\neg(9 / 10$ RemainingF \& $\neg$ NextF). Now, it might be tempting to think the mere fact that $9 / 10$ RemainingF supports NextF shows that $9 / 10$ RemainingF supports $\neg(9 / 10$ RemainingF \& $\neg$ NextF), since NextF entails $\neg(9 / 10$ RemainingF \& $\neg$ NextF). This

${ }^{9}$ Cf. also Steup's (2011: 109-111) Easter Bunny hypothesis. 
kind of line of thought is suggested by Conee \& Feldman (2004: 300-301), also defenders of naïve evidentialism, when they write that "the evidence for ordinary beliefs argues against any proposition that one sees to conflict with the ordinary beliefs, while one has no new reason to doubt those beliefs or their support."

However, this sort of reasoning is fallacious. ${ }^{10}$ Here is a simple counterexample. Suppose Trump, Cruz and Rubio are all running for the Republican nomination. Let $\mathrm{T}$ be the proposition that Trump wins (while Cruz and Rubio do not) and $\mathrm{C}$ be the proposition that Cruz wins (while Trump and Rubio do not). Now suppose you learn that Rubio is dropping out. This evidence supports $\mathrm{T}$ (in the sense that it raises T's evidential probability, compared with before you got this new evidence). But it need not thereby support $\neg$ C, even though $T$ entails $\neg$ C. Indeed, it actively supports $C$. When you learn that Rubio is dropping out, the evidential probability both of $\mathrm{T}$ and of $\mathrm{C}$ increases, even though $\mathrm{T}$ and $\mathrm{C}$ are inconsistent with one another, so that each entails the negation of the other.

The general point here is that evidence in favor of some proposition $\mathrm{p}$ need not tell against every p-alternative; it can often support $\mathrm{p}$ merely by telling against some $\mathrm{p}$-alternatives. Thus, Conee \& Feldman's principle fails. ${ }^{11}$ To come full circle back on the Blackjack case, 9/10RemainingF can support NextF not by telling against the alternative hypothesis that $(9 / 10$ RemainingF \& $\neg$ NextF), but rather by telling against other alternatives to NextF - namely, those where the next card is $a$ number card, but not the last remaining number card out of 10 total remaining cards. And similarly, the appearance of hands might support the proposition that you have hands not by telling against skeptical hypotheses, but rather by telling against other alternatives to the proposition that you have hands namely those where you don't have hands, but there is no great skeptical deception causing it to appear that you do have hands (for example, cases where your hands have been chopped off and you can see this).

But maybe the blackjack case nevertheless shows that some principle that the opponent of the naïve evidentialist is relying on is false. Markosian identifies several such candidate principles, some of which I do not endorse. However, I'll argue that there are the two principles that are (each, individually) enough to secure a refutation of naïve evidentialism, but which the blackjack case is not a counterexample to. Here is a first claim:

Evidential Parity 1. If some candidate evidence $E$ is at least as well-predicted by $\neg p$ as it is by $\mathrm{p}, \mathrm{E}$ cannot support $\mathrm{p}$.

Is the blackjack case a counterexample to Evidential Parity 1? First, I conceded earlier that 9/10RemainingF does support NextF. Does Evidential Parity 1 say otherwise? No, it does not. According to Evidential Party 1, for 9/10RemainingF to support NextF, it must also be the case that

\footnotetext{
${ }^{10}$ Roger White (2006: 232) has pointed this out in another context.

11 As I'll clarify in section (III)b below, the failure of this principle is consistent with thinking that if the all-thingsconsidered probability of $\mathrm{p}$ (given one's total evidence) is above a threshold $\mathrm{T}$, and p entails $\mathrm{q}$, then the all-thingsconsidered probability of q (given one's total evidence) is also above T. The truth of this principle, together with its superficial similarity to the fallacious principle here, may help to explain the apparent intuitive support for the latter. (Thanks to two anonymous referees here.) As should become clear later in the present section, the true principle here is not of help to the naïve evidentialist.
} 
NextF predicts $9 / 10$ RemainingF better than $\neg \mathrm{NextF}$ predicts $9 / 10 \mathrm{Remaining} F$. But that is true: given only the assumption that NextF, the probability that 9/10RemainingF is much higher than it would be given only the assumption that $\neg \mathrm{NextF}$. By contrast, it is true that the hypothesis that (9/10RemainingF \& $\neg \mathrm{NextF}$ ) - the analogue of the skeptical hypothesis - predicts the evidence that 9/10RemainingF just as well as (in fact, better than) the hypothesis that NextF. Indeed, it predicts this evidence trivially, by entailing it. But $(9 / 10$ RemainingF \& $\neg \mathrm{NextF})$ is not the negation of NextF. So Evidential Parity 1 does not say that 9/10RemainingF can't support NextF.

Similarly, Evidential Parity 1 does not say that 9/10RemainingF can't support (9/10RemainingF \& NextF). For (9/10RemainingF \& NextF) predicts 9/10RemainingF better than $\neg(9 / 10$ RemainingF \& NextF) does. Again, the skeptical hypothesis that $(9 / 10$ RemainingF \& $\neg N e x t F)$ predicts $9 / 10$ RemainingF just as well as (9/10RemainingF \& NextF), but again, the former is not the negation of the latter.

One might worry that Evidential Parity 1's focus on comparing a proposition only with its negation is conveniently narrow. Consider:

Evidential Parity 2. If some candidate evidence E is just as well-predicted by some proposition $\mathrm{q}$ as it is by some proposition $\mathrm{p}, \mathrm{E}$ cannot support $\mathrm{p}$ over $\mathrm{q} .{ }^{12}$

Evidential Parity 1, in fact, falls out of Evidential Parity 2. Generally, E can support p in absolute terms without supporting $\mathrm{p}$ over every alternative proposition $\mathrm{q}$ (we saw this earlier with the Trump/Cruz/Rubio example). But in the special case where $q$ is itself the negation of $p$, E cannot support $\mathrm{p}$ without supporting $\mathrm{p}$ over $\mathrm{q}$ : evidence cannot raise the probability of $\mathrm{p}$ without increasing the probability of $\mathrm{p}$ relative to its own negation. Thus, it will follow from E's failing to support p over its own negation that it cannot support p simpliciter. Thus, Evidential Parity 2 entails Evidential Parity 1. Though the reverse entailment does not hold, one might think that Evidential Parity 1 gets its plausibility from the same rationale that motivates the broader Evidential Parity 2 . And now, the naïve evidentialist might say, if Evidential Parity 2 is flawed, and the plausibility of Evidential Parity 1 derives from (the rationale for) Evidential Parity 2, then Evidential Parity 1 is also called into question.

I do indeed endorse Evidential Parity 2, at least with the following interpretative stipulation. In talking of E supporting p "over" q, I mean that E increases the ratio of the probability of the former to the probability of the latter. It does not suffice for E's supporting $\mathrm{p}$ over $\mathrm{q}$, in my sense, that it increases the absolute probability of $\mathrm{p}$ more than it increases the absolute probability of $\mathrm{q} .{ }^{13}$ This will be important later.

\footnotetext{
12 This is close to what Markosian (2014: 172) calls the "Prediction Principle".

${ }^{13}$ It does not matter whether this tracks the ordinary meaning of the phrase 'supporting p over q', so long as the principle as I am stipulating it creates trouble for naïve evidentialism. But, just for the sake of interest, here is why I think it does. Return to the example of Trump and Cruz. Suppose that there is independent evidence that Trump is more likely to win than Cruz. If this is so, then Rubio's dropping out will increase the absolute probability that Trump will win more than it increases the absolute probability that Cruz will win. But this isn't because Rubio's dropping out supports Trump's winning over Cruz's winning, in any ordinary sense; rather, it's all down to the independent evidence that Trump is more likely to win that Cruz. That means that when both their chances go up by the same ratio, Trump's go up more in absolute terms.
} 
Is the blackjack case a counterexample to Evidential Parity 2? Since the analogue of the skeptical hypothesis, (9/10Remaining $\mathrm{F} \neg \mathrm{NextF})$, predicts $9 / 10 \mathrm{Remaining} F$ at least as well as both NextF and (9/10RemainingF \& NextF), Evidential Parity 2 entails that 9/10RemainingF cannot support either of these latter claims over the former. But is that the wrong result? To investigate this, let us draw a diagram which informally models one's belief updating when one learns that 9/10RemainingF. To make things easier, let us suppose that before one learns this, one already knows that either 9 of the 10 remaining cards are face cards, or 9 of the remaining 10 cards are number cards, but has no idea which. So one's prior credence that $9 / 10$ RemainingF is 0.5 , and the remaining credal space is taken up by possibilities where 9 of the remaining 10 cards are number cards. These assumptions are utterly inessential and are made only for conveniently reducing the number of possibilities that we have to represent.

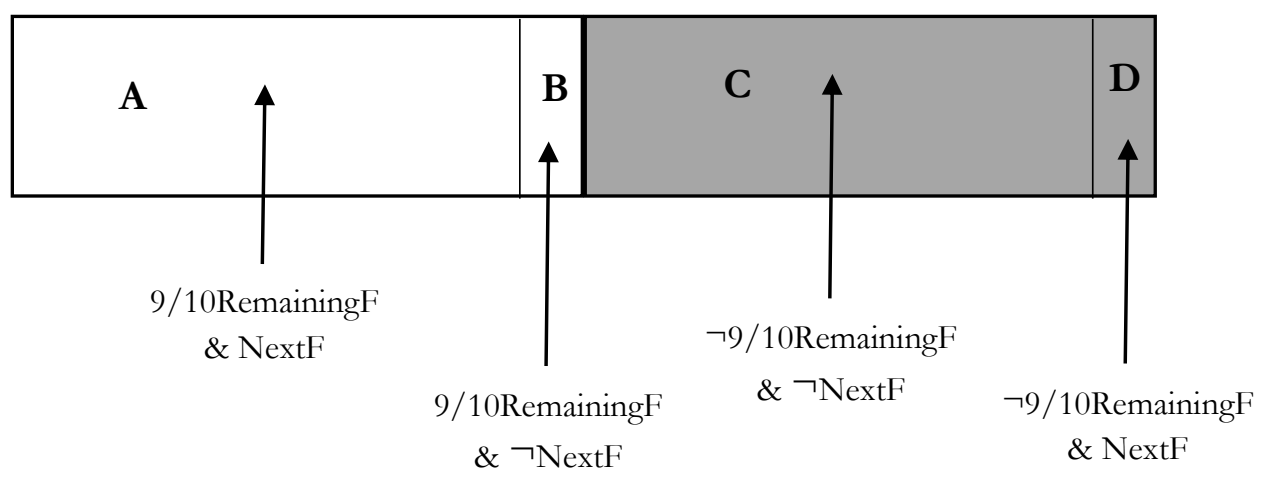

The grey shading represents the elimination of boxes $C$ and D from one's credal space when one learns that 9/10RemainingF. Here are one's credences for the various propositions before and after this update:

\begin{tabular}{|l|l|l|}
\hline & Before & After \\
\hline 9/10RemainingF \& NextF (box A) & 0.45 & 0.9 \\
\hline 9/10RemainingF \& $\neg$ NextF (box B) & 0.05 & 0.1 \\
\hline$\neg 9 / 10$ RemainingF \& $\neg$ NextF (box C) & 0.45 & 0 \\
\hline$\neg 9 / 10$ RemainingF \& NextF (box D) & 0.05 & 0 \\
\hline 9/10RemainingF (boxes A+B) & 0.5 & 1 \\
\hline$\neg 9 / 10$ RemainingF (boxes C+D) & 0.5 & 0 \\
\hline NextF (boxes A+D) & 0.5 & 0.9 \\
\hline$\neg N e x t F$ (boxes B+C) & 0.5 & 0.1 \\
\hline
\end{tabular}

As we can see, and as we conjectured in the discussion of Evidential Parity 1, learning that 9/10RemainingF does support both NextF and (9/10RemainingF \& NextF), in that it raises the probability of these propositions. However, it does not support either of these propositions over the proposition (9/10RemainingF \& $\neg \mathrm{NextF})$, given our ratio-based interpretation of what this means. ${ }^{14}$

14 The evidence does increase the probability of $(9 / 10$ RemainingF $\&$ NextF) by more than it increases the probability of (9/10RemainingF \& $\neg \mathrm{NextF}$ ), in absolute terms. But, as with the Trump/Cruz case (cf. the previous footnote), this is all 
Put another way, it does not change the partition of the credal space between boxes A and B. So there is no violation of Evidential Parity 2 here.

Note that in the same sense, the new evidence does (as it is intuitive to say) support NextF over $\neg \mathrm{NextF}$, as it raises the ratio of the probability of the former to the latter. This is consistent with Evidential Parity 2, however, since, as we noted in the discussion of the Evidential Parity 1, NextF does predict $9 / 10$ RemainingF better than $\neg$ NextF does.

At this point the naïve evidentialist might try a different response. She might concede that 9/10RemainingF does not support (9/10RemainingF \& NextF) - or NextF - over (9/10RemainingF $\& \neg \mathrm{NextF})$. Yet, she might insist, in the blackjack case, one's total evidence supports $(9 / 10 \mathrm{Remaining} F$ \& NextF) over $(9 / 10$ RemainingF \& $\neg \mathrm{NextF}) .{ }^{15}$ Indeed, we can see this, the naive evidentialist might say, from the fact that in our table above, the former has probability 0.9 after the evidential update that is, given one's total evidence - whereas the latter has only probability 0.1 . Yet, both propositions predict even one's total evidence equally well. So if we focus on total evidence, the case does constitute a counterexample to Evidential Parity 2.

In response, begin by noting an often-missed ambiguity in talk of what the total evidence supports. ${ }^{16}$ When we talk of what the total evidence supports, we often mean to refer to the credences that are supported on balance, given one's total evidence. But in talking of what credence is supported given one's total evidence we also encode the antecedent probabilities that the relevant propositions enjoyed before any specific evidence was gathered or learned. On the other hand, one could distinguish a narrower usage of talk of being supported by the total evidence, where the total evidence is the sum of things that one has specifically learnt, and something is supported by the total evidence only if its probability is raised by those items specifically; its being antecedent probable, before any evidence was gathered, does not count as its being supported by the evidence in this sense.

Now, in the blackjack case, it is antecedently likelier that (9/10RemainingF \& NextF) than that (9/10RemainingF \& $\neg \mathrm{NextF}$ ), before any evidence has been gathered. Given what those propositions mean, and how objective chance works, the former is by definition more likely than the latter. It's also true that once specific evidence has been gathered, (9/10RemainingF \& NextF) remains more probable than $(9 / 10$ RemainingF \& $\neg \mathrm{NextF})$. But that is not because any of the evidence gathered supports the former over the latter; it is all due to the former's being antecedently more probable than the latter. So, while there is a sense (the broad sense) in which the total evidence supports the former over the latter, there's no individual piece of evidence that supports the former over the latter; rather, what accounts for the former's being more probable than the latter, having taken into account the total evidence, is its having been antecedently more probable than the latter, before any evidence was gathered. So the total evidence does not support the former over the latter in the narrower sense.

The defender of Evidential Parity 2 should now insist that when Evidential Parity 2 talks of E supporting p over q, it uses 'evidential support' in the narrower sense, referring only to the specific support lent by the totality of the propositions that compose E, and not to the support that $\mathrm{p}$ and $\mathrm{q}$

\footnotetext{
due to the antecedent ratio of the probability of the former to the latter, which remains unchanged after the evidential update.

${ }^{15}$ Cf. Markosian (2014: 172).

16 Thanks to an anonymous referee here.
} 
may have enjoyed antecedently to those proposition been taken account of as part of one's evidence. So the defender of Evidential Parity 2 should concede that it can be the case that, given the antecedent probabilities plus some body of evidence $\mathrm{E}, \mathrm{p}$ is more probable than q, even though q predicts $\mathrm{E}$ just as well as p does. But, she maintains, it cannot be that the propositions composing $\mathrm{E}$ themselves lend support to p over q, even though q predicts $E$ just as well as p does.

Note, moreover, that this concession (if it be such) does not represent a victory for the naïve evidentialist. Returning to the case of anti-skeptical assumptions, what the naive evidentialist holds is that it is the appearance of hands that supports the hypothesis that one has hands over contrary skeptical hypotheses. But the analogue of the appearance of hands in the blackjack case is $9 / 10$ RemainingF. But it has already been shown that 9/10RemainingF does not support (9/10RemainingF \& NextF) (or NextF itself) over ( $9 / 10$ RemainingF \& $\neg \mathrm{NextF})$. What accounts for this is instead, that the former is antecedently likelier than the latter. The analogue of this for anti-skeptical assumptions would be to hold, not that the appearance of hands supports the hypothesis that one has hands over contrary skeptical hypothesis, but that something else makes the former antecedently likelier than the latter, before such appearances have been taken into account. ${ }^{17}$ And that view is not naive evidentialism; indeed, it is just what the naïve evidentialist is concerned to deny. (That said, it is a possible view, and we'll consider it in section III(c).) So, the game was in fact already up when our hypothetical naïve evidentialist conceded that $9 / 10$ RemainingF does not support (9/10RemainingF \& NextF) over ( $9 / 10$ RemainingF $\& \neg \mathrm{NextF})$. In fact, this is so irrespective of whether switching to talk of total evidence impugns Evidential Parity 2 (which I've suggested, on a charitable reading of Evidential Parity 2, it does not.)

I conclude that the blackjack case does not impugn the parity principles. The other cases fail to do so for similar reasons. Yet the parity principles do rule out the naïve evidentialist position. For the appearance of hands to support believing that one is not a brain in a vat (of a suitably refined kind) would be a violation of Evidential Parity 1 (and, since all violations of Evidential Parity 1 are violations of Evidential Parity 2, also Evidential Parity 2). After all, the hypothesis that you're a brain in a vat having hand-hallucinations predicts the evidence that it appears you have hands at least as well as the hypothesis that you're not a brain in a vat having hand-hallucinations. ${ }^{18}$

Although I have not given any knockdown argument for the evidential parity principles, they are prima facie plausible, and I have suggested that this plausibility withstands Markosian-style attacks. So, we have a strong reason for worrying about whether ordinary evidence can support anti-skeptical assumptions.

\section{(b) Epistemic priority}

The other broad group of reasons for thinking of anti-skeptical assumptions as taken on without evidence concerns the idea that they are in some sense foundational or prior to our ordinary beliefs and judgments about our evidence for those beliefs. In some sense, it can seem that the belief that one has

\footnotetext{
${ }^{17}$ Cf. White 2006: 552-3.

${ }_{18}$ There is, by the way, an analogous point to be made in the blackjack case. Given the parity principles, the evidence that $9 / 10$ RemainingF cannot support the analogue of the anti-skeptical assumption, namely $\neg(9 / 10$ RemainingF \&

$\neg \mathrm{NextF}$ ). As our table above shows, however, this is the right result: the probability of the that anti-skeptical assumption actually goes down given the evidence that $9 / 10$ RemainingF.
} 
hands is based on the assumption that things are roughly as they seem, rather than the other way round.

The point here can be put as a challenge for those who think that ordinary appearances count as evidence for anti-skeptical assumptions. It seems clear that there are certain cases where some belief is powerless to support another belief, even though the first belief entails the second. To take an example from Keith DeRose (2000: 704-5), suppose I believe the Cubs beat the Cardinals solely based on my having read this in the newspaper. ${ }^{19}$ From my belief that the Cubs beat the Cardinals and that the newspaper reported this, I could deduce that the newspaper reported the score rightly. But it seems odd to think that I could ever come to be justified this in forming this latter belief this way. After all, in some sense, I only believe the Cubs beat the Cardinals because I am already assuming that the newspaper was reporting the score rightly. ${ }^{20}$ Intuitively, when I read the newspaper report, I don't learn anything new about the newspaper's reliability when I read its claim that the Cubs beat the Cardinals, if I have no independent corroboration for this claim. If I did, then as Stewart Cohen (2002, 2010) points out, I could repeat this process again and again to come to believe, inductively, that the newspaper is very accurate quite generally, thus "bootstrapping" my way to a belief about the newspaper's track-record. So it seems that, in this case, the proposition that the Cubs beat the Cardinals cannot support the proposition that the newspaper is reliable.

The challenge for someone who thinks that ordinary appearances can support anti-skeptical assumptions is to explain what is disanalogous about that structure compared with this newspaper case. Intuitively, there are commonalities: in inferring that I have hands from the appearance of hands, it seems that I am (at least implicitly) already assuming that perception is reliable, i.e. that I am in the good case and that anti-skeptical assumptions do not hold. This again seems to limit the role that ordinary appearances can play as evidence for anti-skeptical assumptions. Moreover, in the newspaper case, one happily does in fact typically have further, independent background empirical evidence that newspapers tend to report sports scores reliably. But when it is the basic tie between appearance and reality that is in question - when the skeptical hypothesis is global rather than local - it is not clear what evidence could play a role in justifying anti-skeptical assumptions without already taking their truth for granted. ${ }^{21}$

Relatedly, anti-skeptical assumptions can seem so foundational to our cognitive systems as to be things that are taken for granted or on faith without evidence. ${ }^{22}$ They are, to be somewhat vague, so foundational that nothing can be said in their favor without begging the question. The whole enterprise of taking things to be evidence for other things cannot get off the ground until such assumptions are

\footnotetext{
${ }^{19}$ See also Cohen $(2002,2010)$ for related cases.

${ }^{20}$ Note that the idea here isn't most naturally developed as being the radically closure-violating idea that although I'm justified in believing that the Cubs beat the Cardinals, and justified in believing that the newspaper reported this, I'm not justified in believing that the newspaper is correct. Rather, in the spirit of closure, the idea is more naturally developed as the point that I have to have already been (at least propositionally) justified in believing that the newspaper was reliable if I am to justifiably form the belief that the Cubs beat the Cardinals when I read the report.

${ }^{21}$ Steup (2011: 113) suggests that we have plenty of background evidence against the existence of brains in vats - that they don't appear in textbooks on neurophysiology, and so on. But surely all of this could be explained away by a suitably detailed skeptical hypothesis - whereas the general reliability of the newspaper is not severely called into question by the hypothesis that it reported today's score wrongly.

22 This sort of position is often associated with Wittgenstein (1969).
} 
made. This may suggest a special role for anti-skeptical assumptions that somehow explains why they have a distinctive status in being able to be rationally held without evidence, and without taking oneself to have evidence.

\section{A priorism}

The foregoing arguments put pressure on the idea that anti-skeptical assumptions are typically held on the basis of ordinary empirical evidence. But perhaps such anti-skeptical assumptions are known $a$ priori, antecedently to our ordinary knowledge. ${ }^{23} \mathrm{I}$ will subdivide this view into three categories. To set up this subdivision, recall from section II(a) that we can distinguish different ways of talking of what the evidence supports. On a narrower interpretation, to say that a proposition is (adequately) supported by the evidence is to say that the evidence one has learnt (or updated on) has raised the proposition's probability; on a broader interpretation, it is to say that given the total evidence, the probability of the proposition is (adequately) high, where the probability also reflects any pre-evidential antecedent likelihood that the proposition enjoyed.

On the first version of the a priori view that I will consider, the a priori justification for antiskeptical assumptions is understood as constituting evidence for them, such that the anti-skeptical assumptions are supported by the total evidence in both the narrow and the broad sense. On the second version of the view, the a priori justification is understood as not affecting the probability of anti-skeptical assumptions, either by evidentially raising their probability or by making them more preevidentially probable - such that they are supported by the total evidence neither in the narrow nor the broad sense. On the third version of the view, the a priori justification is understood as making them pre-evidentially probable, such that they are supported by the total evidence in the broad but not the narrow sense.

\section{(a) A priori justification as evidence}

Begin with the first option. The mere a priori status of someone's justification need not rule out its counting as evidential in a suitably broad sense. Those who favor a priori accounts of mathematical, logical and indeed (at least a large portion of) philosophical knowledge have not always thought of such beliefs as lacking any kind of evidential grounding. On many prominent accounts, a priori justification is to be understood in terms of a priori intuitions or seemings that evince the truth of propositions, thereby counting as evidence for those propositions. ${ }^{24}$

One might hope to extend this idea to anti-skeptical assumptions. If one is to hold that antiskeptical assumptions are known or justified a priori, it would be good to be able to tell some story about why such assumptions count as being a priori justified. One potential answer is that what justifies us in believing that we're not brains in vats is the fact that this hypothesis seems so bizarre to us. If we reject skeptical hypotheses partly because they seem bizarre to us, we seem to be treating the

\footnotetext{
${ }^{23}$ This view is developed in different ways by Cohen (2010), DeRose (forthcoming: esp. ch. 7), White (2006), and Wright (2004).

${ }^{24}$ See, e.g., Bealer (2000).
} 
apparently bizarreness as an indicator of its falsity; that is, as evidence against the skeptical hypothesis and in favor of its negation.

The problem with this way of making sense of the a priori view, however, is that it seems to be vulnerable to the same problems surveyed in the previous section for empirical evidence. As Descartes himself notes in the Meditations, ${ }^{25}$ we can build into our skeptical hypothesis that, just as empirical appearances are not a good guide to reality, so too our a priori seemings are not a good guide to reality. In fact, once one lets the seeming that one is not a brain in a vat count as an a priori seeming, this and other similar a priori seemings will be unreliable in skeptical scenarios as they stand. After all, when one is in a skeptical scenario, the skeptical hypothesis will still seem bizarre. So it is part of the skeptical hypothesis that one has a priori seemings as of being in the good case. As a result, the same, Evidential Parity-generated objections apply equally to the view on which one has a priori evidence that one is not a brain in a vat.

The problem here is a more general one for a priori knowledge of (deeply) contingent truths, provided one tries to construe this knowledge as being secured by evidence. The paradigmatic candidates for a priori knowledge are beliefs in necessary truths. Since there simply are no worlds in which these necessary truths are false, we can sustain the idea that we possess a priori evidence for such beliefs in all possible worlds, without countenancing violations of the parity principles. When it is a belief in a contingent truth that is at issue, things are not the same. Unless one can somehow have a priori seemings that actually track contingent truths - and it's a bit hard to see how such intuitions would then be a priori - the a priori seeming would appear to be no better predicted by the truth of the contingent proposition than by its falsity. And that leads us into violations of the parity principle.

More arguably, the present view may also be subject to the objections from the previous section concerning epistemic priority. If we grant that it is built into a skeptical hypothesis that one's a priori seemings are also out of whack with the world, then in basing one's belief in an anti-skeptical assumption on an a priori seeming, one seems also to be already taking for granted that one's a priori seemings are reliably tied to the truth such that they can serve as evidence. But this is to, in effect, already assume that the anti-skeptical assumption fail to hold.

(b) A priori justification as probabilistically irrelevant

The second category of views includes views on which our justification for believing anti-skeptical assumptions is robustly pragmatic, where pragmatic reasons to believe anti-skeptical assumptions are ones that do not themselves bear on the likelihood of the truth of those assumptions, either evidentially or in some pre-evidential way. ${ }^{26}$ It also includes views on which some less paradigmatically pragmatic factor - such as our finding skeptical hypotheses bizarre, or the lack of simplicity of skeptical hypotheses - is taken to justify us in believing them, but not to make them more likely to be true (again, either evidentially or in some pre-evidential way). ${ }^{27}$

\footnotetext{
${ }^{25}$ Descartes (1641/1996: 14-15).

${ }^{26}$ Cf., e.g., Rinard (ms.), and on some readings, Wright (2004).

${ }^{27}$ DeRose (forthcoming: ch. 7) might be an example of this view, though he might be closer to the view of the next subsection; he does not explicitly distinguish the two views.
} 
Clearly, proponents of such views must reject the evidential requirement, which requires that we not believe anything for which we lack adequate evidence. Moreover, it seems that any reflective agent who follows the present view's advice in a self-aware manner would have to violate the interlevel coherence (ILC) principle, since she would recognize herself as believing something without evidence. Let us consider what such an agent would really have to be like.

To believe that $\mathrm{p}$ is to believe that $\mathrm{p}$ is true. But one cannot coherently believe that $\mathrm{p}$ is true without also (at least implicitly) believing that $\mathrm{p}$ is, all-things-considered, likely to be true. (I'll use 'likely' here to refer to any probability greater than $50 \%$.) So, to be coherent, someone who believes anti-skeptical assumptions must also believe that such assumptions are likely to be true. But the view that we are currently considering, recall, says both (i) that there is nothing that makes anti-skeptical assumptions antecedently likely to be true, before the evidence is taken account of and (ii) that we lack evidence that raises the likelihood of anti-skeptical assumptions from their pre-evidential level. Moreover, there can't be something non-evidential that raises the likelihood of anti-skeptical assumptions from their pre-evidential level, since anything that raises the likelihood of $\mathrm{p}$ from its preevidential level just is, ipso facto, evidence for $\mathrm{p}$. From this, it follows that a reflective agent who believes and follows the view that we are presently considering is also committed to thinking that it is not the case that anti-skeptical assumptions are likely to be true.

So, this agent is committed both to thinking that anti-skeptical assumptions are likely to be true and to thinking that it's not the case that anti-skeptical assumptions are likely to be true. This lays bare the incoherence of such a position. Such a combination of attitudes cannot be rational; indeed, I doubt whether it is even possible for a reflective agent who is conscious of her own mental states. ${ }^{28}$ (Notice that the argument I've given is not just an argument against the view we are currently considering, but a defense of (ILC), when interpreted using the broader notion of evidential support.)

An alternative option for someone who thinks that anti-skeptical assumptions are not supported by the evidence, even in the broad sense, might be to concede to the skeptic that we are not justified in believing anti-skeptical assumptions. Nevertheless, this view says, anti-skeptical assumptions are justified qua assumptions, where assumption is taken seriously as a sui generis kind of mental state distinct from belief. ${ }^{29}$ Perhaps it is part of what is distinctive of such a cognitive status that it can be held without evidence, and acknowledged as such. Note that such cases are not violations of (ILC) if assumption is not taken to be a doxastic state, but some non-doxastic cognitive propositional attitude.

However, there is another problem with the view that anti-skeptical assumptions are not supported by the evidence in the broad sense, that applies whether the appropriate attitude towards such propositions is understood as belief or as a sui generis state of assumption. As skeptics so often exploit, ordinary propositions (such as the proposition that one has hands) entail anti-skeptical assumptions. But it is a simple axiom of probability that, where p entails $\mathrm{q}$, the probability of p cannot exceed the probability of $\mathrm{q}$. Thus, if one says that anti-skeptical assumptions are not probable on the evidence, even in the broad sense of this locution, then one must either concede that ordinary propositions are also not probable on the evidence, or countenance violations of this simple axiom of

${ }^{28}$ See Worsnip (forthcoming-b) for more on this latter point.

${ }^{29}$ Views of this sort are suggested by Wright (2004: 175-8); Sherman \& Harman (2011); Pritchard (2016). 
probability. But adherents of the sui generis assumptions view do not want to hold that "ordinary" propositions about the external world can only (justifiably) be assumed, rather than believed. Rather, they want to hold that these ordinary propositions can be (justifiably) believed, despite "resting" on assumptions that are only justified qua assumptions. Thus, they are committed to violations of the axioms of probability in the structure of evidential support.

Crispin Wright, a defender of the sui generis assumptions view, explicitly holds that evidential support is not closed under logical entailment, which one might take to constitute a biting of the bullet on this point. But when he comes to explaining how this is so, he appeals to the point that some particular body of evidence E can support p but not support q, even though p entails $q .{ }^{30}$ I have already agreed with that claim in the discussion of naïve evidentialism in section II(a); it was illustrated by the Trump/Cruz/Rubio example. But crucially, it doesn't follow from this that one's total evidence can support $\mathrm{p}$ to some degree $\mathrm{N}$ without supporting q to at least degree $\mathrm{N}$, where p entails $\mathrm{q}$, and (crucially) where 'evidential support' is interpreted in the broader way that reflects antecedent, pre-evidential likelihood. ${ }^{31}$ Indeed, the same probabilistic framework that debunks the former claim vindicates the latter.

(c) A priori justification as pre-evidential probability

Let us finally turn to the view on which anti-skeptical assumptions do enjoy a pre-evidential, antecedent likelihood. ${ }^{32}$ This view might also be thought of as challenging (ILC). But that is only accurate if (ILC) is interpreted using the narrower notion of evidential support. A defender of this view can admit the truth of (ILC) when interpreted using a broader notion of evidential support. Moreover, the defender of this view can argue (ILC) must be interpreted in this way, in order to take account of cases like the Blackjack case discussed in section II(a) above. Consider, in that case, the analogue of the anti-skeptical assumption, namely $\neg(9 / 10$ RemainingF \& $\neg$ NextF). As we saw in our discussion, this proposition is antecedently probable, before any specific evidence is taken account of, but there is no specific piece of evidence that raises its probability (in fact, the only candidate piece of evidence, 9/10RemainingF, actually lowers its probability, albeit perhaps not low enough such that it is no longer adequately probable to be fit to be believed). Yet it seems perfectly coherent for one to be aware of all this and nevertheless to believe this proposition. ${ }^{33}$ According to the present version of $a$ priorism, anti-skeptical assumptions should be treated correspondingly: they should be regarded as antecedently, pre-evidentially probable.

However, this does inevitably raise the question of in virtue of what anti-skeptical assumptions are antecedently, pre-evidentially probable. Here the proposition $\neg(9 / 10$ Remaining $F \& \neg N e x t F)$ seems rather different from an anti-skeptical assumption. It's enough to show that this proposition is antecedently, pre-evidentially likely to show that if it's true that 9/10RemainingF, then it's unlikely that

\footnotetext{
30 As Wright notes, this is the minimal lesson of Dretske's original attempted counterexamples to closure.

31 And it's fair game to interpret it that way in the present context, since we're considering a view on which anti-skeptical assumptions are not supported by the evidence even in the broader sense.

32 Cf. White (2006: 553).

${ }^{33}$ If it seems to you incoherent to believe something that you assign only credence 0.9 to, simply adjust the example to increase the ratio of remaining face cards to number cards.
} 
$\neg \mathrm{NextF}$, since the only way the proposition as a whole could be false would be if it were both true that 9/10RemainingF and true that NextF. And by the very meanings of the propositions in question, if it's true that 9/10RemainingF, then (assuming, as we should, that we have not yet conditionalized on any other evidence that might be gathered), it's likely that NextF, and thus unlikely that $\neg$ NextF. So (9/10RemainingF \& $\neg \mathrm{NextF}$ ) is antecedently improbable, and its negation is antecedently probable. Thus, simple mathematical reasoning can explain why $\neg(9 / 10$ Remaining $\mathrm{F} \& \neg \mathrm{NextF})$ is antecedently, pre-evidentially probable. But obviously, no parallel reasoning is available to explain why the antiskeptical assumption that (for example) you're not a brain in a vat is antecedently, pre-evidentially probable.

I don't want to insist that there's nothing the a priorist can say here about why anti-skeptical assumptions are antecedently, pre-evidentially probable. But no neat demonstration of this claim, of the kind available in the Blackjack case, is in the offing; it isn't built into the very meaning of the antiskeptical assumption that it is mathematically probable. This at least limits the leverage that the former case has to render plausible a parallel treatment of the latter. It seems too much of a just so story to simply claim that it's a brute fact that anti-skeptical assumptions are probable; something must make them probable. Yet the a priorist (of the kind we're currently considering) also faces the challenge of articulating something that makes it probable, yet doesn't ultimately collapse into a putative piece of evidence for anti-skeptical assumptions, thus landing us back with the problems of the version of $a$ priorism considered in section III(a). (For example: why would the simplicity of a hypothesis make it preevidentially probable, rather than constituting a piece of evidence for its truth against the background of a universe where simple hypotheses tend to be true more often that false ones?) As I said, these challenges may not be insurmountable, but their scale should at least motivate us to look for a less mysterious account.

One much less mysterious route for the a priorist to take is to embrace a much more subjective form of Bayesianism. On this view, any probabilistically coherent set of priors is rational, and it isn't an objective fact that anti-skeptical assumptions are pre-evidentially probable (here, notice, a disanalogy with the Blackjack case is conceded). Rather, anti-skeptical assumptions "having" a high prior probability is simply a matter of our in fact assigning them a high prior probability; anti-skeptical assumptions have a high prior probability for us as creatures who find them antecedently plausible, and taking such an attitude toward them is permissible but not required. However, on this ultrapermissivist view, there is nothing distinctively rational about high priors for anti-skeptical assumptions as opposed to high priors for, say, the proposition that the moon is made of cheese or that the world is ruled by disguised lizards. Since any probabilistically coherent priors are OK, high priors in antiskeptical assumptions are $\mathrm{OK}$, but only vacuously so. This will be of no help or comfort to a reflective agent wondering about whether she should assign a high prior probability to the claim that she is not a brain in a vat.

\section{Externalism}

We are in a bad place. As we saw in sections II and III(a), the idea that anti-skeptical assumptions receive support from our ordinary empirical evidence is subject to some serious objections. But 
equally, as we saw in sections III(b) and III(c), the idea that they can be justified or warranted without evidence is also subject to serious objections. If anti-skeptical assumptions cannot be justified either with or without evidence, and we do not want to be closure-deniers, skepticism about both justified belief and knowledge loom.

A point about the dialectic with the skeptic is in order here. At the outset, I said that the status of anti-skeptical assumptions can be interesting even if one is not moved by skeptical arguments. I stand by this, but I also believe that the considerations surveyed thus far give us genuine reasons to be worried by skeptical arguments. A familiar and simple argument for skepticism moves from the claim that you don't know you're not a brain in a vat, plus a closure principle, to the claim that you don't know you have hands (or any other ordinary empirical proposition). Those who are bullish about skepticism, and some who are not, are often unmoved by this argument for two reasons. First, they often say that they simply don't find the first premise compelling. ${ }^{34}$ For some, it is supposed to be intuitive in a self-standing way. ${ }^{35}$ But even if one doesn't find it intuitively compelling on its own, parts 2 and 3 of this paper can be seen as an argument by cases for this first premise. You don't know that you're not a brain in a vat through ordinary empirical evidence; nor do you know that you're a brain in a vat a priori (understood either evidentially or non-evidentially). But those are the only candidate ways to know that you're not a brain in a vat. So you don't know that you're not a brain in a vat. This fills in a positive case for the first premise in the anti-skeptical argument.

Secondly, some complain that the simple argument doesn't obviously generalize from knowledge to justified belief. ${ }^{36}$ Even if one does find the first premise intuitively compelling for knowledge, one may not find it intuitively compelling for justified belief. But again, the case built up here for the first premise does generalize to justified belief. And it does not just question whether we have sufficient justification for believing anti-skeptical assumptions, where this sufficiency is a matter of meeting some (perhaps quite high) threshold. Rather, we have been questioning whether we have any justification at all, whether evidential or non-evidential, for anti-skeptical assumptions. The problems raised affect any claim that such assumptions amount to justified beliefs. So correspondingly, given a plausible closure principle for justification - or indeed, the probability axiom for total evidential support broadly construed, just mentioned at the end of the last section - the argument as a whole generalizes too, threatening the conclusion that our ordinary beliefs enjoy any justification, or evidential probability, at all.

Thus, while I can't force those who are bullish about skepticism to take this argument seriously, I believe that it is worth doing so.

There may be a chink in the argument's armor, however. In arguing in section II that ordinary evidence cannot support anti-skeptical assumptions, we assumed that one's evidence is the same in the good case and the bad case. This is why any putative evidence that is supposed to favor the good case hypothesis is predicted just as well by the bad case hypothesis, and thus cannot really count in favor of the good case hypothesis, according to the parity principles.

\footnotetext{
34 Pryor (2000: 522); Conee \& Feldman (2004: 300). See Pryor (2000: 527-8) for his own formulation of what he takes to be the most effective skeptical argument.

${ }^{35}$ Cf., e.g., DeRose (1995).

36 Again, see Pryor (2000: 523).
} 
However, the assumption that one's evidence is the same in the good case and the bad case is not universally accepted. An externalist account of evidence rejects this assumption, holding that one's evidence differs cross the two cases. ${ }^{37}$ There is not room here for a detailed assessment of the independent merits of internalism versus externalism about evidence. ${ }^{38}$ Rather, having seen that the internalist account has difficulty delivering evidence for anti-skeptical assumptions, my focus will be on seeing how far the externalist account can go in providing an adequate treatment of them. However, I do want to briefly engage the sometimes-voiced idea that externalism simply changes the subject from what we are ordinarily talking about when we talk about evidence: that the ordinary concept of evidence is just obviously internalist in character.

It's true that some philosophers have taken it for granted that evidence is to be understood in internalist terms. As theories of knowledge and of justification, "internalism" and "evidentialism" were once often used as synonyms. ${ }^{39}$ In what might be characterized as a gradual "externalist turn" in epistemology over the last half-century, it was externalist accounts of knowledge, and then justification, that sprung up first. ${ }^{40}$ To the extent that a theory made knowledge or justification a matter of external reliability, it was initially taken to divorce knowledge or justification from evidence - on the assumption that evidence was to be understood in internalist terms. Only later were externalist accounts of evidence developed.

However, this historical pattern is rather odd, since there is a quite clear externalist strain in ordinary talk about evidence ${ }^{41}$ - indeed, arguably a much clearer strain than is present in talk of justification, or still less of rationality. Suppose Layla believes that Djibouti is a city in Argentina, on the basis of Martin's testimony. And suppose that later Layla discovers that Martin is a compulsive liar, though she had no previous way of knowing this. It's pretty natural for Layla to now say, "well, I thought I had good evidence that Djibouti is a city in Argentina. But it turns out that I didn't. Martin is a compulsive liar: his testimony counts for nothing!" But this remark is very much in the spirit of externalism about evidence. ${ }^{42}$ Layla seems to be taking the actual reliability of Martin's testimony to bear on the quality of evidence that it provided for her belief. This is so even though Layla had no way of knowing at the time that Martin's testimony is generally unreliable, and even though the situation she was in was phenomenally indistinguishable from one in which his testimony is generally reliable. Note that her remark is quite different from her saying, "I did have good evidence that Djibouti is a city in Argentina. But now I know that Martin is a compulsive liar, that evidence has been defeated, and I no longer have good evidence that Djibouti is a city in Argentina." Rather, she takes

\footnotetext{
${ }^{37}$ Such accounts are developed, in various different ways, by McDowell (1995), Williamson (2000), Neta (2003) and Pritchard (2016).

${ }^{38}$ For a particularly detailed and ingenious case for externalism about evidence, see Williamson (2000: chs. 4, 8, 9).

39 The paradigm exponents of the two as a unified view being Conee \& Feldman (1985).

${ }^{40}$ Even after Williamson's seminal work, many philosophers simply write as if internalism about evidence is unquestionably true. For example, Steup (2011: 109) flatly states: "a person who undergoes envatment does not undergo any change of her evidence." Tellingly, even amongst those who are externalists about justification, internalism about evidence is still frequently taken for granted. See, e.g., Zalabardo (2005: 40-2) and Wong (2009: 366).

41 See also Przyjemski (2017: 190-3).

42 Note, however, that interestingly, it seems much less natural for her to say, "well, I thought my belief that Djibouti is a city in Argentina was rational. But it turns out that I wasn't. Martin is a compulsive liar: his testimony counts for nothing!" This suggests that externalist talk about rationality comes less naturally to us than externalist talk about evidence, and that our talk about the two may come apart. See also Worsnip (2016: 358).
} 
herself never to have had good evidence for this proposition, even though it seemed to her that she did.

Let us now turn to what the externalist account says about anti-skeptical assumptions. As noted above, by denying that one's evidence supervenes on phenomenally accessible appearances, the externalist makes room for the possibility that one's evidence differs importantly between the good case and the bad case. However, the externalist still owes us some reason to think that one's evidence actually does differ importantly between the good case and the bad case. There are two different stories that the externalist can tell here. The distinctness and availability of each story becomes clearer when we focus on a relatively simple but oft-neglected distinction between two factors that jointly determine what one's evidence supports. First, there is the question of what one's evidence is: which items make it into one's body of evidence. Secondly, there is the question of what the evidential support relations are between the items in one's body of evidence (even holding that body of evidence fixed) and various potential beliefs. As I understand the externalist, she can hold that the answer to either of these questions can vary according to whether one is in the good case or the bad case.

The more obvious, and oft-explored, thought is that what one's evidence $i$ depends upon whether one is in the good case. On a well-known "disjunctivist" account of perceptual experience, ${ }^{43}$ veridical experience has a different character to non-veridical experience, even when the two are not phenomenally distinguishable. In the good case, one sees that one has hands, where 'sees' is a factive verb. In the bad case, it merely appears that one has hands (even though this is phenomenally indistinguishable from seeing that one has hands). The trick now is to say that this difference affects what one's evidence is. Perhaps when one sees that one has hands, it is then, at least typically, part of one's evidence that one has hands. By contrast, when it merely appears that one has hands, one's evidence is only that one apparently has hands. It's not hard to see why this would affect what the evidence supports believing. Whereas apparent hands are equally well-predicted by the good case hypothesis and the bad case hypothesis, one's actually having hands is only predicted by the former. Thus, if it really is part of one's evidence in the good case that one has hands, this can count as evidence for anti-skeptical assumption without any violation of evidential parity. So, this view says, if one is actually in the good case, then one's evidence supports believing anti-skeptical assumptions (including the very proposition that one is in the good case).

Some will balk at this disjunctivism. This makes it worth acknowledging a second potential externalist story about how one's evidence importantly differs in the good case and the bad case. On this view, even if we characterize what one's evidence is the same way in the good case and the bad case, the facts about what it supports differ in the two cases. ${ }^{44}$ This is especially easy to see with respect to ordinary propositions like the proposition that one has hands. In the good case, things are generally as they appear to be. In the bad case, they are not. This means that appearances are a much more reliable guide to reality in the good case than they are in the bad case. So, much as the reliability of Martin's testimony can (on the externalist account) affect its evidential value, the reliability of

\footnotetext{
${ }^{43}$ Especially associated with McDowell $(1982,1995)$. See Logue (2013) for a compelling recent defense of disjunctivism against some standard worries.

${ }^{44}$ Byrne and Logue (2008) draw a related, but perhaps not quite equivalent, distinction between "metaphysical" and "epistemological" disjunctivism.
} 
appearances can affect their evidential value. In the good case, the appearance of hands is better evidence for the proposition that one has hands than it is in the bad case. The difference between the cases here comes in not in what one's evidence is but in what (even given a fix on what it is) it supports.

What about an anti-skeptical assumption, like the proposition that one is not a brain in a vat, or the very proposition that one is in the good case? One might worry that if we say that the appearance of hands is evidence for this anti-skeptical assumption, even only in the good case, we are still led into a violation of the parity principles. After all, were one in the bad case, one would still have the appearance of hands as part of one's evidence. But the externalist may be able to reply here. On the externalist view, fixing what one's evidence is does not on its own fix all the salient properties of one's evidence. One's evidence has the property of supporting certain propositions, and that can depend on external facts about reliability as well as what one's evidence is. Given that, perhaps the parity principles should be interpreted so that, for there to be evidential parity between $\mathrm{p}$ and not-p, it must be not only that not-p predicts what evidence one has just as well as p does, but that not-p predicts all the properties of one's evidence just as well as p does. But, on the externalist account (unlike the internalist account), were a skeptical hypothesis true (and so an anti-skeptical assumption false), one's evidence would not have the same supporting properties that it does. So, if one is in the good case, one has evidence with properties that are not predicted by the proposition that one is in the bad case. The externalist can claim that this is enough to disrupt evidential parity. Thus, this externalist can maintain that when one is in the good case (but not when one is in the bad case), appearances support anti-skeptical assumptions over their negations.

We now have two potential ways for the externalist to develop her account: either by maintaining, with the disjunctivist, that what one's evidence is depends on whether one is in the good case, or by maintaining, with a kind of reliabilist about evidential support, that what one's evidence supports depends on whether one is in the good case. Either account, I have suggested, allows the externalist to maintain that anti-skeptical assumptions are supported by one's ordinary evidence without allowing violations of the parity principles. On either account, such anti-skeptical assumptions are only evidentially supported when one actually is in the good case.

\section{A hybrid view}

We could stop here, and many externalists do. However, there are two things that should cause us to still feel some dissatisfaction as things stand. First, the questions about epistemic priority raised in section II(b) remain. There is a lingering sense that anti-skeptical assumptions are somehow foundational or groundless, and that ordinary appearances are ill-suited to ground them. A full account would ideally have something to say to do justice to this sense. Second, some do not find the externalist account very reassuring. On this account, we may have evidence that we are in the good case - but only if we actually are in the good case. The impetus behind the internalist account may be to find a kind of justification that is available whether or not we are in the good case - some reason for taking ourselves to be in the good case that is not conditional on our actually being so.

I suggest that these two sources of dissatisfaction are highly related. Let us make the following stipulative definition: 
Radically question-begging evidence (definition). Some state $\mathrm{S}$ is radically questionbegging evidence for $\mathrm{p}$ iff S's counting as evidence for $\mathrm{p}$ itself depends on p's being true.

Note that on both ways of developing externalism above, the evidence for anti-skeptical assumptions is radically question-begging in this sense. On the disjunctivist view about what evidence one has, the appearance as of hands amounts to seeing that one has hands when one is in the good case, but to a mere appearance as of hands when one is in the bad case. Only in the former case (assuming evidential parity) is it evidence for the proposition that one is in the good case. On the reliabilist view about support, the mere appearance as of hands is evidence for the proposition that one is in the good case when one actually is in the good case, but not in the bad case. In both cases, then, one has only radically question-begging evidence for $\mathrm{p}$.

Note that the same is not true of the support for ordinary propositions like the proposition that one has hands itself. Even on the externalist account, the mere appearance of hands can still be evidence that one has hands even when one does not have hands, and so it is non-question begging evidence that one has hands. This is no violation of evidential parity, because the bare hypothesis that it is not the case that one has hands does not predict the appearance of hands as well as the hypothesis that one does have hands does. The externalist account (at least in its reliabilist version) does say that the mere appearance of hands is not such good evidence that one has hands when one is in the bad case. But not all scenarios in which one lacks hands are ones where one is in the bad case, that is, a victim of a global deception such that appearances are a generally poor guide to reality. And as long as the appearance of hands is evidence that one has hands in some cases where one does not have hands, it is not radically question-begging evidence.

I suggest that the externalist can say that the apparent groundlessness of anti-skeptical assumptions is explained by the fact that all our evidence for them is radically question-begging. The only considerations that support the proposition that one is in the good case do so only if one actually is in the good case. So in this sense, in citing them as evidence that one is in the good case, one is already presupposing that one is in the good case.

One could make either a weaker or a stronger claim here. The weaker claim is that radically question-begging evidence for $\mathrm{p}$ is merely psychologically unsatisfying as a ground for believing $\mathrm{p}$. The stronger claim is that radically question-begging evidence for $\mathrm{p}$ is a normatively inappropriate ground for believing $\mathrm{p}$. Though both claims represent a concession to the animating spirit behind internalism, the second makes more of a concession than the first. Against the internalist, it still holds that there can be radically question-begging evidence for $\mathrm{p}$ that is still properly speaking evidence for $\mathrm{p}$; but it agrees with the internalist that this is an inappropriate ground on which to base one's belief in p. ${ }^{45} \mathrm{I}$ will not take a stance on one should opt for this stronger claim or merely for the weaker, psychological, claim. Either way, we have an explanation of the sense of groundlessness of anti-skeptical assumptions,

\footnotetext{
45 This option requires one to concede that there can be evidence one has, and that supports a proposition, but that it is inappropriate to base one's belief on. Some will find that a prohibitive cost to the second option (in which case, the first remains a fallback), but I myself do not.
} 
as well as of the sense that there is a problem of epistemic priority. On the present diagnosis, the problem is not after all that ordinary assumptions simply cannot be evidence for anti-skeptical assumptions - for the externalist, that will just be settled by whether one actually is in the good case. But it is to say that in taking oneself to have ordinary evidence for anti-skeptical assumptions, one already presupposes those that those anti-skeptical assumptions are true.

The flip-side of this, however, is that, if one is an externalist (whether explicit or tacit) about evidence, and one believes that one is in the good case, then along with this belief will naturally come a belief that one has evidence that one is in the good case. After all, that's what the externalist account says about one's situation in the good case. So, as long as one believes that one is in the good case, and one is a tacit externalist, one can also be expected to believe that one's evidence supports believing that one is in the good case. This means that one will satisfy inter-level coherence. Notice that this is the case regardless of whether one actually is in the good case or not. For the externalist, if one is unluckily in the bad case, one's beliefs will then unluckily fail to match one's actual evidence: but one's belief-set will still be entirely understandable and coherent. Again, this is a partial concession to the internalist account. Whether one satisfies coherence requirements like (ILC) is a matter solely of one's (non-factive) attitudes and not of whether the external world co-operates. Consequently, we can get a sort of internalist normative notion that won't be susceptible to standard externalist attacks: that of rational coherence, in the broad sense of conforming to (ILC) and other requirements like it. ${ }^{46}$

One may think that an agent's merely satisfying (ILC) - that is, avoiding a simultaneous position of believing $\mathrm{p}$ while believing herself to lack adequate evidence for $\mathrm{p}-$ is not of much significance. But I think that it is actually of crucial significance. A key apparent problem with the proposal that we can or should believe anti-skeptical considerations for non-evidential reasons is that it leaves us violating (ILC), in a position of synchronic incoherence. However, the present account suggests that anti-skeptical assumptions are a special case. On the externalist account, anti-skeptical assumptions are unusual in that they tend to bring along with them (if one is at least a tacit externalist) the belief that they themselves are evidentially supported. Once we believe the anti-skeptical assumption, we can conclude that we have evidence for the anti-skeptical assumption.

So perhaps we should take a second look at the view that we might in some sense come to believe anti-skeptical assumptions for non-evidential reasons. On the version of that view considered in section III(b), we can rationally do this even though it will leave us violating (ILC). I rejected that version of the view. However, if we instead marry the view with externalism about evidence, we do not need to say this. Instead we can say the following: even if one comes to believe an anti-skeptical assumption for a non-evidential reason, in this special case, the belief can then bring along with it the higher-order belief that it is supported by the evidence. ${ }^{47}$ And so, unlike most cases of trying to believe

\footnotetext{
46 The kind of argument given by Williamson (2000) attempts to show, in effect, that no normative epistemic notion will be construable in internalist terms. But this, I believe, overlooks the possibility of a normative notion framed in terms of the satisfaction of coherence requirements that are wide-scope in the sense identified in section I. While such a notion will not, I think, be “luminous" in Williamson's sense, it will nevertheless both supervene on non-factive mental states and resist being understood in terms of knowledge.

${ }^{47}$ One might make an objection here. Above I entertained the view that because evidence for anti-skeptical assumptions is radically question-begging, it is inappropriate to base one's belief in such anti-skeptical assumptions on this evidence. If this is so, then even if we can permissibly (and correctly!) take ourselves to have evidence for anti-skeptical assumptions, we cannot permissibly base our belief in such anti-skeptical assumptions on this evidence. And this might be thought in
} 
for non-evidential reasons, it will not cause one to violate (ILC). That is why it is possible to respond to non-evidential reasons in these cases without irrationality or incoherence.

Of course, more would have to be said about the character of the non-evidential reasons in question. Perhaps we can make use of the idea that anti-skeptical assumptions are needed in order to get one's cognitive projects off the ground or for them to stand any chance of succeeding. ${ }^{48}$ This is not the place to try to execute this task in any detail. My point here is that the current account clears the ground for a version of it that would not embroil us in incoherence, where we believe without taking ourselves to have any evidence. This can be achieved, I have suggested, by marrying such an approach with externalism about evidence.

Note that, in a final concession to the spirit of internalism, the non-evidential reasons to take oneself to be in the good case would obtain both in the good case and in the bad case, and would thus avoid question-begging status. Effectively, they would be a license to believe oneself to be in the good case, and thus, to start taking appearances to be evidence, both for ordinary propositions and for antiskeptical assumptions themselves. Note, however, that on the externalist account, this need not be some kind of pretense. Rather, provided that one is in the good case, one will be perfectly correct to take appearances to be evidence, and one's practices will line up with reality just so. It's only in the bad case that things will be out of whack. But we knew that many things were going to be out of whack with each other in the bad case. Why shouldn't this just be one more instance of that?

Of course, few people have explicit views about evidential externalism. But I do not think the present account is obviously a long way from our actual practice. We do assume that things are roughly as they seem to be, and we do assume that ordinary appearances support our ordinary beliefs. Once we make this foundational assumption, we will take ourselves to be believing things for evidential reasons, not for pragmatic reasons. But we will struggle to present a non-question-begging evidential reason for the assumption itself. We make it, in some sense, to get our whole procedure of evidential assessment off the ground.

The strategy we have arrived at is a relatively complex hybrid. ${ }^{49}$ Let me conclude by summarizing it. First, we make use of externalism about evidence to show that we can have ordinary evidence for anti-skeptical assumptions, as long as those anti-skeptical assumptions are actually true. By the same token, we show how we can coherently take ourselves to have evidence for anti-skeptical

itself problematic. In particular, it might suggest that even if we have propositional justification for anti-skeptical assumptions, we lack doxastic justification for them, at least of an epistemic kind. (Thanks to an anonymous referee here.) I cannot do full justice to this set of questions here, but let me make a few quick points. First, even if we lack doxastic justification of an epistemic kind for anti-skeptical assumptions, I still think it is a significant result to show that reflective agents who believe anti-skeptical assumptions for pragmatic reasons need not violate (ILC) when it comes to antiskeptical assumptions. A pure non-evidential strategy of the kind considered in section III(b) does not deliver this result. I am more committed to (ILC) than I am to the evidential requirement, due to the argument given for (ILC) in that section. Secondly, I did not firmly endorse the view that it is inappropriate to base beliefs on question-begging evidence; I noted the alternative position that it is merely psychologically unsatisfying to do so. This view does not have any results about (lack of) doxastic justification. Thirdly, one can imagine a compromise view here, on which it is inappropriate to come to believe anti-skeptical assumptions on the basis of question-begging evidence, but once one already believes these assumptions, and draws the conclusion that there is also evidence for them, one may permissibly base one's belief (in whole or in part) on this evidence.

48 See Wright (2004) and especially Enoch \& Schechter (2008).

49 Pritchard's (2016) view is also a kind of hybrid of an externalist view and a view involving non-evidential entitlement. But the view he ends up at is nevertheless quite different from mine. 
assumptions, and thus avoid synchronic incoherence. But the view recognizes that our taking ourselves to have this evidence in some way presupposes the truth of the anti-skeptical assumptions in question, and so is not a satisfying ground for this belief. At that point, non-evidential reasons enter the picture. Normally, such non-evidential reasons are difficult to respond to without incoherence, since doing so typically involves believing while taking oneself not to have evidence. But the special character of anti-skeptical assumptions means that in this case this problem does not apply. If we are tacit externalists about evidence, our belief in anti-skeptical assumptions will bring along with it a belief that we have evidence for anti-skeptical assumptions. This means that we can come to believe them for non-evidential reasons, without being left in a position where we believe something for which we take ourselves to lack evidence, nor indeed (if we are in the good case) something for which we actually lack evidence.

The account sketched here has been programmatic, but it promises several important virtues over its rivals. Unlike the internalist version of the view that we have ordinary evidence for antiskeptical assumptions, it avoids violations of the evidential parity principles that I defended. Unlike the view that anti-skeptical assumptions are made without evidence of any kind, it avoids embroiling us in synchronic incoherence. Yet unlike externalism about evidence on its own, it explains why such anti-skeptical assumptions feel (and perhaps in some sense are) evidentially groundless. Moreover, while embracing externalism about evidence, it does justice to various internalist impulses, including that to provide some non-question-begging consideration in favor of believing anti-skeptical assumptions that is, considerations that apply both in the good case and in the bad case. No doubt some will feel that this combination of features is too good to be true. But I hope that they are tantalizing enough at least to entice them into articulating why this should be so. In the meantime, I hold out some hope that this just might be a case where we can have the best of all worlds.

\section{References}

Bealer, G. (2000). 'A Theory of the A Priori,' Pacific Philosophical Quarterly, 81/1: 1-30.

Broome, J. (1999). 'Normative requirements,' Ratio, 12: 398-419.

(2013). Rationality Through Reasoning. Chichester: Wiley-Blackwell.

Byrne, A. \& Logue, H. (2008). 'Either/Or,' in Haddock \& Macpherson (eds.), Disjunctivism: Perception, Action, Knowledge. Oxford: Oxford University Press.

Cohen, S. (2002). 'Basic Knowledge and the Problem of Easy Knowledge,' Philosophy and

Phenomenological Research, 65/2: 309-329.

------- (2010). 'Bootstrapping, Defeasible Reasoning, and A Priori Justification,' Philosophical

Perspectives, 24: 141-159.

Conee, E. \& Feldman, R. (2004). 'Making Sense of Skepticism,' in their Evidentialism. Oxford:

Oxford University Press.

Dancy, J. (1977). 'The logical conscience,' Analysis, 37/2: 81-84.

DeRose, K. (1995). 'Solving the Skeptical Problem,' Philosophical Review, 104/1: 1-52.

(2000). 'Ought We to Follow Our Evidence?,' Philosophy and Phenomenological Research, 60/3: 697-706.

------ (forthcoming). The Appearance of Ignorance. Oxford: Oxford University Press. 
Descartes, R. (1641/1996). Meditations on First Philosophy, trans. Cottingham. Cambridge, UK: Cambridge University Press.

Enoch, D. \& Schechter, J. (2008). ‘How Are Basic Belief-Forming Methods Justified?’ Philosophy and Phenomenological Research, 76/3: 547-579.

Greco, D. (2012). Epistemic Levels. Dissertation, Massachusetts Institute of Technology.

Horowitz, S. (2014). 'Epistemic Akrasia,' Noûs, 48/4: 718-744.

Logue, H. (2013). 'Good News for the Disjunctivist about the Bad Cases,' Philosophy and

Phenomenological Research, 86/1: 105-133.

Markosian, N. (2014). 'Do You Know that You Are Not a Brain in a Vat?,' Logos \& Episteme, 5/2: 161-181.

McDowell, J. (1982). 'Criteria, Defeasibility, and Knowledge,' Proceedings of the British Academy, 68: 455-479.

(1995). 'Knowledge and the Internal,' Philosophy and Phenomenological Research, 55/4: 877-893.

Neta, R. (2003). 'Contextualism and the Problem of the External World,' Philosophy and Phenomenological Research, 66/1: 1-31.

Pritchard, D. (2016). Epistemic Angst. Princeton: Princeton University Press.

Pryor, J. (2000). 'The Skeptic and the Dogmatist,' Noûs, 34/4: 517-549.

Przyjemski, K. (2017). 'Strong Epistemic Possibility and Evidentiality,' Topoi.

Rinard, S. (ms.). 'Pragmatic Skepticism,' draft manuscript.

Scanlon, T.M. (2007). 'Structural Irrationality,' in Brennan, Goodin, Jackson \& Smith (eds.), Common Minds: Themes from the Philosophy of Philip Pettit. Oxford: Oxford University Press.

Sherman, B. \& Harman, G. (2011). 'Knowledge and Assumptions,' Philosophical Studies, 156/1: 131-

140.

Steup, M. (2011). 'Evidentialist Anti-Skepticism,' in Dougherty (ed.), Evidentialism and its Critics.

Oxford: Oxford University Press.

White, R. (2006). 'Problems for Dogmatism,' Philosophical Studies, 131/3: 525-557.

Williamson, T. (2000). Knowledge and its Limits. Oxford: Oxford University Press.

Wittgenstein, L. (1969). On Certainty (eds. Anscombe \& von Wright). Oxford: Blackwell.

Wong, W. (2009). 'Internalism about justification and the skeptic's dilemma,' Erkenntnis, 71/3: 361375.

Worsnip, A. (2016). 'Moral reasons, epistemic reasons, and rationality,' Philosophical Quarterly, 66/263: 341-361.

(forthcoming-a). 'The conflict of evidence and coherence,' Philosophy and Phenomenological Research.

(forthcoming-b). 'What is (in)coherence?,' Oxford Studies in Metaethics.

Wright, C. (2004). 'Warrant for Nothing (and Foundations for Free)?,' Proceedings of the Aristotelian Society Supplementary Volume, 78: 167-212.

Zalabardo, J.L. (2005). 'Externalism, Skepticism, and the Problem of Easy Knowledge,' Philosophical Review, 114/1: 33-61. 